\title{
Miranda
}

Revue pluridisciplinaire du monde anglophone /

Multidisciplinary peer-reviewed journal on the English-

speaking world

$17 \mid 2018$

Paysages et héritages de David Bowie

\section{Paysages berlinois et héritage romantique : les univers culturels et sonores de la trilogie Low-'Heroes'-Lodger (1976-1979)}

\section{Hugues Seress}

\section{(2penEdition}

\section{Journals}

Édition électronique

URL : http://journals.openedition.org/miranda/13101

DOI : 10.4000/miranda.13101

ISSN : 2108-6559

\section{Éditeur}

Université Toulouse - Jean Jaurès

\section{Référence électronique}

Hugues Seress, «Paysages berlinois et héritage romantique : les univers culturels et sonores de la trilogie Low-'Heroes'-Lodger (1976-1979) », Miranda [En ligne], 17| 2018, mis en ligne le 20 septembre 2018, consulté le 16 février 2021. URL : http://journals.openedition.org/miranda/13101 ; DOI : https:// doi.org/10.4000/miranda.13101

Ce document a été généré automatiquement le 16 février 2021.

\section{cc)}

Miranda is licensed under a Creative Commons Attribution-NonCommercial-NoDerivatives 4.0 International License. 


\title{
Paysages berlinois et héritage romantique : les univers culturels et sonores de la trilogie Low-'Heroes'- Lodger (1976-1979)
}

\author{
Hugues Seress
}

\section{Introduction}

L'antagonisme fécond entre académisme et avant-garde fournit aux arts musique, peinture, littérature, cinéma -, une richesse de langage, et les révolutions esthétiques nécessaires à la perpétuation de leur histoire. L'académisme défend le respect des conventions formelles que l'avant-garde s'évertue à déconstruire, en défrichant de nouveaux espaces d'expression qui, par leur assimilation progressive, redéfinissent les modèles à suivre. Les influences réciproques entre ces deux démarches démontrent leur profonde interdépendance: sans académisme, pas d'avant-garde et inversement. Si le modernisme considère l'innovation comme annonciatrice des normes de demain, le postmodernisme abolit cette hiérarchie par un jeu d'influences croisées, tant dans la temporalité et la géographie des esthétiques, que dans la sociologie des cultures ${ }^{1}$.

1 Ces quelques mots, qui pourraient concerner de nombreux parcours d'artistes modernes et postmodernes, d'ailleurs pas uniquement rock, sont ceux que Mathieu Thibault dédie à David Bowie dans l'hommage qu'il lui rend au sein d'un ouvrage paru en $2013^{2}$. Dans cet ouvrage, l'auteur considère la démarche du « compositeur-peintreacteur-interprète " comme profondément sous-tendue par la notion de "collection d'images de pensée $»^{3}$, correspondant à l'ère d'un temps de fragmentation culturelle et sociale. En effet, tout au long de sa carrière d'artiste, et peut-être plus particulièrement dans sa période dite "berlinoise ", Bowie manifeste cette tendance à l'emprunt et à l'hybridation de matériaux et d'images d'origines historiques et culturelles multiples, volontairement dissonants pour créer un objet global, dont la signification dépasse chacune des références prises séparément. 
2 Cette période commence, d'une certaine manière, dès l'hiver 1975-1976, et se poursuit par la tournée Station to Station durant le printemps 1976 : elle emmène David Bowie et Iggy Pop à travers l'Europe, de gare en gare, pour la promotion de l'album éponyme. L'artiste sort d'une période difficile, celle des albums Ziggy Stardust et de Young Americans, les dernières années aux États-Unis et notamment à Los Angeles l'ayant mené au bord de l'épuisement et de la schizophrénie, dans une sorte de perception virtuelle de lui-même, qu'ont accentuée la composition d'alter ego protéiformes et la prise de drogues dures.

Un livre, Mr. Norris Changes Train (1935) de l'écrivain britannique Christopher Isherwood qu'il rencontre sur la route en février 1976, le convainc définitivement de s'établir dans la capitale allemande. Le roman conforte le fantasme pour l'époque révolue de l'entre-deux-guerres de Bowie, qui imagine Berlin comme un lieu de profonde animation culturelle lui offrant l'anonymat nécessaire à une nouvelle vie débarrassée des excès de Los Angeles ${ }^{4}$.

3 La réduction au silence, à une forme de sobriété de l'expression, s'impose donc comme une réaction salutaire à la surenchère glamour de la période précédente. Bowie déclare : «Lorsque j'ai quitté Los Angeles, j'ai découvert que je connaissais si peu de choses, que j'avais très peu à dire. Le manque de paroles dans l'album Low montre que j'étais littéralement à court de mots $»^{5}$. La mise en scène du personnage du Thin White Duke en transit dans les gares allemandes, scandinaves et britanniques est souvent interprétée au-delà des intentions mêmes de l'artiste. Bowie se défend d'être un sympathisant fasciste et justifie l'utilisation d'une imagerie référentielle quelque peu ambiguë à des fins théâtrales. Il affirme :

Non, non, non... Je suis Pierrot, je suis comme tout le monde. Ce que je fais est du théâtre, et seulement du théâtre. Je m'utilise moi-même comme une toile et essaie de peindre sur moi la vérité de notre temps. Le visage pâle, les pantalons à pattes d'éléphant - c'est Pierrot, le clown éternel qui dissimule la grande tristesse de $1976^{6}$.

4 En matière musicale également, se dessine une tendance à la sobriété : les matériaux prennent la forme, dans un premier temps, de cellules rythmiques répétées en boucle et de successions simples et mécaniques d'accords triadiques, Ces accords, souvent joués aux synthétiseurs, sont choisis en fonction des propriétés de leur enchaînement. Ils ont une capacité au statisme et, simultanément, permettent une sortie rapide de l'espace diatonique. "L'inquiétante étrangeté $»^{7}$ qui se dégage des ambiances musicales reflète directement les expérimentations du rock électronique alors en vogue en Allemagne dans les années 1970. C'est précisément ce qui intéresse Bowie à une époque où lui-même est en proie à un tiraillement profondément humain, entre les excès d'une vie déformée et rendue virtuelle par l'usage des psychotropes et l'aspiration à se reconnecter à une spiritualité organique.

En 1975 et 1976, il [Bowie] est fou de groupes allemands [...] .Bowie est de nouveau libre de se concentrer uniquement sur ce qui l'intéresse réellement: l'art. Le funk américain et la soul ne sont plus d'actualité [...]. Il en a assez de Los Angeles, de l'Amérique, du rock and roll [...]. La musique électronique allemande est la nouveauté la plus radicale que la pop music a à offrir à cette période ${ }^{8}$.

5 Ainsi, si la période dite "berlinoise» de Bowie a été initiée par une profonde dépression personnelle et stimulée par l'attrait d'un nouvel univers musical existant depuis le début des années 1970 au centre du continent européen, elle ne se réduit cependant pas uniquement à un phénomène psychologique fortuit. Elle ne résulte pas uniquement d'une rencontre entre la subjectivité d'un artiste déjà complet et 
l'objectivité d'un lieu, à la fois chargé de références historiques complexes et d'expériences avant-gardistes contemporaines. Parce que les figures tonales et harmoniques utilisées dans la trilogie Low-'Heroes'-Lodger ${ }^{9}$ constituent la résultante des sèmes culturels présents dans les temps et les espaces auxquels les titres se réfèrent, cet article propose de décliner ces sèmes ${ }^{10}$, afin de saisir le lien existant entre ces formes musicales et le contexte qui les a générées. En remontant de la scène Krautrock allemande à l'ambiance de la ville au passé protéiforme, j'analyserai ce qui sous-tend le choix de Bowie de s'installer et d'investir Berlin comme le lieu privilégié d'un emprunt à l'histoire passée et contemporaine de la ville. Je tenterai de montrer comment l'artiste y puise la culture des hommes qui y vivent, mais aussi celle de ceux qui y ont vécu et qui, par apports successifs, ont nourri la ville de leurs existences respectives.

\section{L'Allemagne : la scène Krautrock et l'émergence de la Cold Wave}

6 Bowie se tourne vers l'Allemagne dès 1975. Dans l'interview qu'il donne pour le magazine Uncut, l'artiste revient sur le contexte musical de son installation dans la capitale allemande. Il évoque l'importance qu'a eue la sortie de l'album Autobahn de Kraftwerk ${ }^{11}$ sur l'évolution de sa pensée et de sa pratique musicale durant cette époque. L'utilisation des instruments électroniques au sein d'univers minimalistes et mécaniques entièrement maîtrisés :

Leurs compositions étaient bien préparées et polies avant d'entrer dans le studio. Mon travail tendait vers des pièces d'ambiance expressionniste, le protagoniste (moi-même) s'abandonnant à l'air du temps (un terme populaire à l'époque), avec peu ou pas de contrôle sur sa propre vie. Leur musique était spontanée pour la plupart et créée dans le studio [...]. Ce qui me passionnait chez Kraftwerk, c'était leur détermination particulière de se tenir loin des grilles d'accords américaines stéréotypées et l'empathie pour la sensibilité européenne qui se manifestait dans leur musique. Ils ont eu une très grande influence sur moi ${ }^{12}$.

7 Même s'il reconnaît que le groupe Kraftwerk a joué un rôle important au moment de la création de l'album Station to Station, Bowie précise que la sortie de Trans-Europe-Express (1977) n'a pu constituer une source directe de son inspiration, parce qu'elle est de quelques mois ultérieure à celle de Station to Station (1976). C'est d'ailleurs de la démarche musicale de Neu $!^{13}$ que l'artiste se sent le plus proche. Il semble défendre davantage une conception plus improvisée de la composition en studio. La rencontre avec la musique de Neu ! semble avoir constitué un choc plus direct, plus émotionnel. Dans le même interview, il ajoute :

J'ai acheté mon premier vinyle Neu 2 à Berlin autour de 1975, lors d'une brève visite. [...] Je l'ai acheté parce qu'ils constituaient un dérivé de Kraftwerk, et qu'ils étaient très intéressants à écouter. En vérité, ils devaient prouver qu'ils étaient les frères anarchistes et rebelles de Kraftwerk. J'ai été complètement séduit par le son agressif des drones de guitare contre la batterie robotique quasiment, mais pas entièrement, calme de Dinger. Bien qu'elle soit assez atténuée, vous pouvez percevoir un peu de leur influence sur la piste Station to Station ${ }^{14}$.

8 C'est bien au-delà du strict musical que l'on peut définir le phénomène Krautrock allemand. Ce dernier constitue un complexe transversal à de nombreuses expressions artistiques : la peinture, l'architecture, la littérature, le cinéma et la mode. Citant Hugo Wilcken, dans son ouvrage concernant l'album Low, Rüther écrit : 
Bowie, comme Kraftwerk, concevaient leur création comme un tout - la musique, le look vestimentaire, l'œuvre d'art [...] complètement intégrés et autoréférentiels [...] tous deux nourrissaient un domaine sensible en travaillant le lien entre ironie et sérieux. Les deux mêlaient le pastiche moderne et l'esthétique rétro. Tous deux faisaient de la musique émotionnelle tout en simulant de nier l'émotion. Vue au travers du prisme de la psychiatrie, l'œuvre des deux artistes apparaissait plutôt comme autiste ${ }^{15}$.

9 Les expériences de métissage entre les musiques électroniques naissantes, le rock et la musique contemporaine rendent singulière la nouvelle vague de groupes qui émerge dès le début de la décennie: Kraftwerk, Neu !, Can ou encore Tangerine Dream. Cependant, l'hétérogénéité stylistique et musicale des groupes allemands de l'époque est en réalité bien plus large que ce qui est perçu de l'étranger, particulièrement des États-Unis. C'est d'ailleurs bien cette hétérogénéité qui intéresse Bowie dans le rock allemand: l'énorme socle d'expressions sonores comme reflet d'un non moins large socle de références culturelles et artistiques. Depuis Station to Station, Bowie cherche à réaliser une hybridation du langage du Rhythm and Blues et de l'univers sonore de la musique européenne. La découverte des vinyles Autobahn de Kraftwerk et Neu 2 en 1975, la rencontre avec les membres de Kraftwerk en 1976 à la gare de Düsseldorf, le souvenir évoqué de cette rencontre dans l'album Trans-Europe-Express de 1977, constituent autant de témoignages de cette quête et de l'engouement de Bowie pour l'Allemagne.

En plus des contestations estudiantines qui traversent l'ensemble du monde occidental de l'époque, l'Allemagne possède des spécificités : elle est confrontée à une époque clé de son histoire, qui prépare le nouveau rayonnement international de sa culture. Ce renouveau est réalisé par une nouvelle génération, confrontée à une dénazification réelle du pays, pour laquelle les simples mots de "grandeur " et de "culture » allemandes pourraient apparaître suspects. Pour ce faire, cette nouvelle génération réutilise les mots, les signes ou les références constitutifs du fascisme, non pour en faire l'apologie, mais pour le dénoncer et s'en débarrasser définitivement. C'est ainsi que, par exemple, l'homme machine de Kraftwerk, reflet du surhomme froid, initié par le futurisme italien et relayé par le nazisme allemand comme antidote à la faiblesse et à l'erreur humaines, semble-t-il présenter simultanément des tendances aussi contradictoires qu'une ode à la technique et une réaction à ses excès. Il est curieux de constater que l'on trouve ces mêmes contradictions, inhérentes à l'homme moderne, dans le socle idéologique néo-romantique du fascisme: apologie du modernisme hyperrationnel et réaction organiciste.

11 Il est difficile de réaliser une synthèse des caractères du langage rythmique, harmonique et instrumental des groupes Krautrock allemands, tant leur hétérogénéité stylistique semble large. On peut toutefois identifier quelques constantes : étirement du temps jusqu'au statisme grâce au format inhabituel des morceaux et à l'élargissement du rythme harmonique, étrangeté et onirisme des ambiances sonores se manifestant notamment dans les enchaînements d'accords et des tonalités, ainsi que dans le choix des timbres (mélange des instruments acoustiques et des synthétiseurs). Le titre emblématique de Kraftwerk, Trans-Europe-Express propose, par exemple, une alternance de deux pôles harmoniques Em (celui du refrain) et $\mathrm{C} \# \mathrm{~m}$ (celui du couplet), à distance de tierce mineure descendante. La relation entre les deux triades de ces tonalités constitue une variation de la relation générique $R$, dite Relative, ( $\mathrm{EM}-\mathrm{C} \# \mathrm{~m}$ ), à laquelle s'ajoute un changement de mode (Em - EM). 
Fig. 1
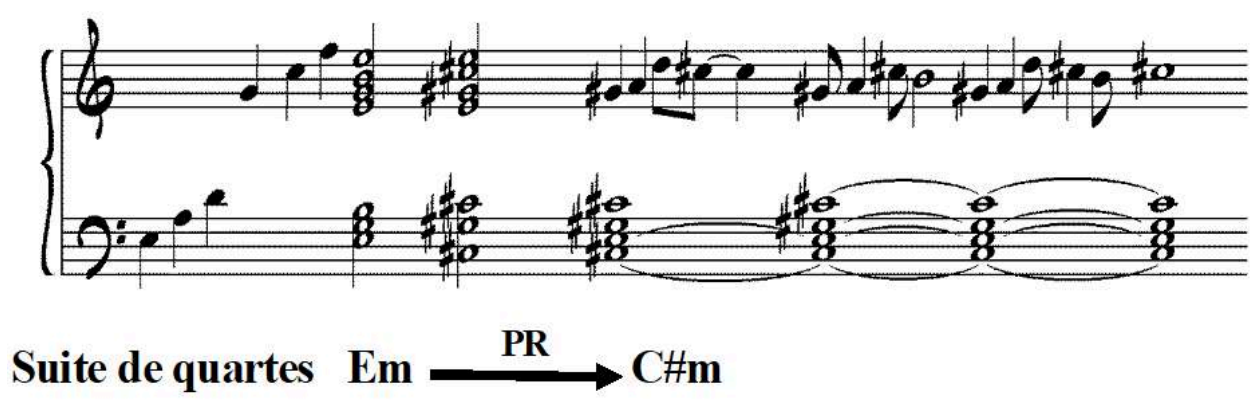

Enchaînement harmonique principal de T.E.E. de Kraftwerk

Ainsi que le soutiennent les théories néo-riemanniennes ${ }^{16}$, les relations harmoniques couplant une distance de tierce entre les fondamentales et un changement de mode (majeur vers mineur ou mineur vers majeur) se développent considérablement dans la musique romantique et postromantique du XIX ${ }^{e}$ siècle, car elles permettent d'échapper à un discours tonal fondé strictement sur la fonction syntaxique et la résolution de la tension harmonique. En outre, comme pour une transformation néo-riemannienne ( $\mathrm{R}$ Relative, L Leadingtone exchange, $\mathrm{P}$ Parallel), la rétention des hauteurs de la triade est maximale (deux des trois hauteurs sont maintenues) ou la parcimonie minimale (la troisième hauteur se déplace d'un demi-ton ou d'un ton ascendant ou descendant), il en résulte un effet de suspension et de statisme harmonique, et simultanément d'une perte de la polarité tonale. Si la transformation est précédée, comme c'est le cas pour le titre de Kraftwerk cité plus haut, d'un changement de mode (Em - EM - C\#m), un chromatisme survient dans l'une des trois voix de la succession d'accords et il s'ensuit une sensation d'étrangeté, consécutive à la sortie de l'espace diatonique (sol-sol\# pour notre exemple).

Fig. 2

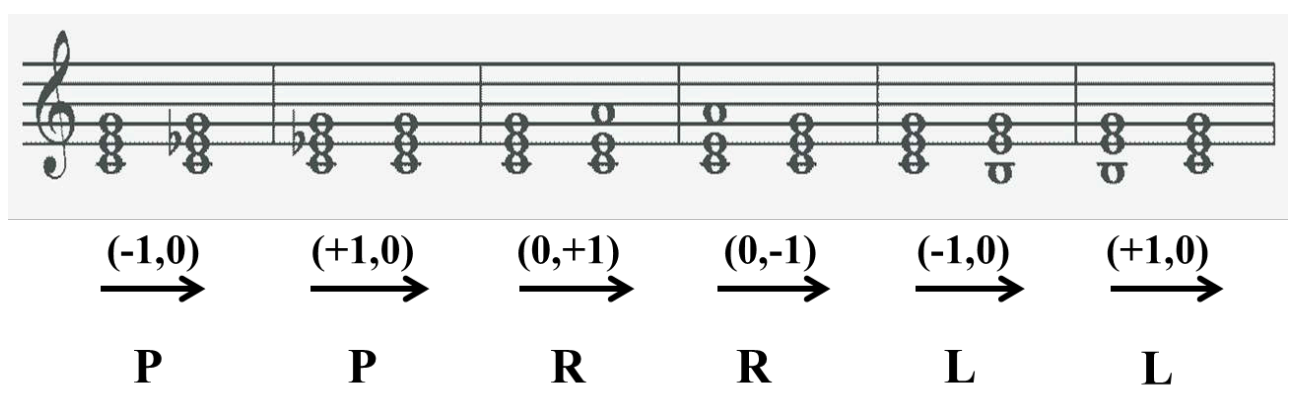

Transformations néo-riemanniennes élémentaires ${ }^{17}$

Ainsi que le soutient Richard Cohn, théoricien américain traitant de la relation de l'univers sonore harmonique et d'un monde suggéré, étrange et onirique

les progressions chromatiques excitaient l'imagination romantique, parce qu'elles ne se conformaient pas aux attentes du comportement et de la succession des triades, mais précisément parce qu'elles les brouillaient [...]. même dans la musique instrumentale non programmatique, elles sont capables d'évoquer l'étrange, le magique et l'insondable ${ }^{18}$. 
Cette tendance à l'étrangeté de l'harmonie se manifeste également dans le rock et les musiques amplifiées, particulièrement à partir du milieu des années 1970 dans le Krautrock allemand, préparant le déferlement de la Cold Wave des années 1980.

On peut relever de nombreux exemples de ce type de relations harmoniques dans la musique de Kraftwerk, de Neu !, et des autres groupes allemands de l'époque. Ces caractères harmoniques ont probablement exercé une influence non négligeable sur la composition de Bowie dans sa période berlinoise, mais il serait erroné de soutenir qu'il n'en existe pas d'exemples avant 1976. Dès la fin des années 1960, alors que le rock est en pleine phase psychédélique, "Space Oddity ${ }^{19}$, de 1969, offre un de ces enchaînements caractéristiques CM-Em, qui, associé à un état de suspension et de statisme avant le décollage de la fusée du Major Tom, confère à ce début de chanson une atmosphère toute particulière, à la fois irréelle et profondément mélancolique.

Fig. 3

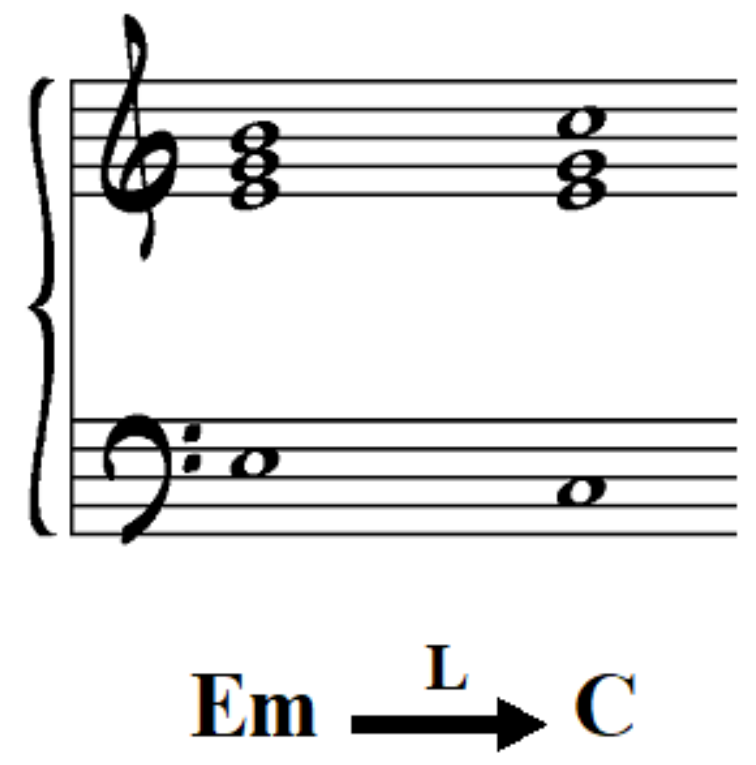

Enchaînement harmonique de Space Oddity

Après Berlin, on trouve également, mais en nombre plus important, ce type de relation harmonique. Plus de dix ans plus tard, l'exemple de «Scary Monsters $»^{20}$ (1980), extrait de l'album éponyme, présente pour le solo, dans une couleur étrange et un timbre proche de la Cold Wave britannique naissante ${ }^{21}$, une succession harmonique, Dm-Bm, composée d'une relation $\mathrm{P}$ suivie d'une relation $\mathrm{R}$ : la même que celle utilisée par Kraftwerk dans son titre Trans-Europe-Express de 1977. 
Fig. 4

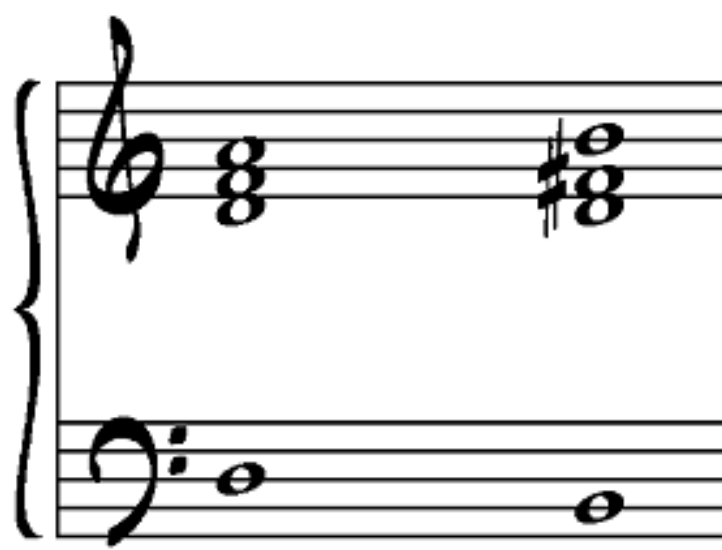

\section{$\mathrm{Dm} \stackrel{\mathrm{PR}}{\longrightarrow} \mathrm{Bm}$}

Enchaînement harmonique de Scary Monsters

17 Dans l'émergence des esthétiques post-punk, la Cold Wave occupe une place particulière. Elle se caractérise par une prédominance des timbres électroniques au service d'ambiances irréelles et oniriques.

Autoproclamé non-musicien, Brian Eno, s'emploie dès la fin des années soixante à bousculer les règles établies entre musique classique écrite et enregistrement rock. Il développe un usage empirique du studio d'enregistrement, considérant les bandes magnétiques comme matériau musical sujet aux traitements de postproduction, montage, boucle, variation de vitesse, effets divers, etc... ${ }^{22}$.

L'importance de l'influence de Brian Eno sur l'évolution musicale, et plus particulièrement harmonique, de Bowie n'est plus à démontrer. Selon Rüther, les deux hommes poursuivent le même objectif : « Eno souhaite faire fusionner ce truc rigide et étrange appelé Kraftwerk, avec le feeling des groupes funk américains. "Lorsque tu trouves quelqu'un avec les mêmes problématiques", dit Eno, "tu as tendance à devenir ami avec lui" " $»^{23}$. L'instrumental "Warszawa $»^{24}$ (1976), extrait de l'album Low, le premier de la trilogie qui initie la collaboration entre David Bowie et Brian Eno doit, pour une part non négligeable, son étrange couleur, intemporelle, à la fois caractéristique de cette période du rock européen, aux successions et aux glissements harmoniques à distance de tierce mineure, de triton et de changement de mode. 
Fig. 5
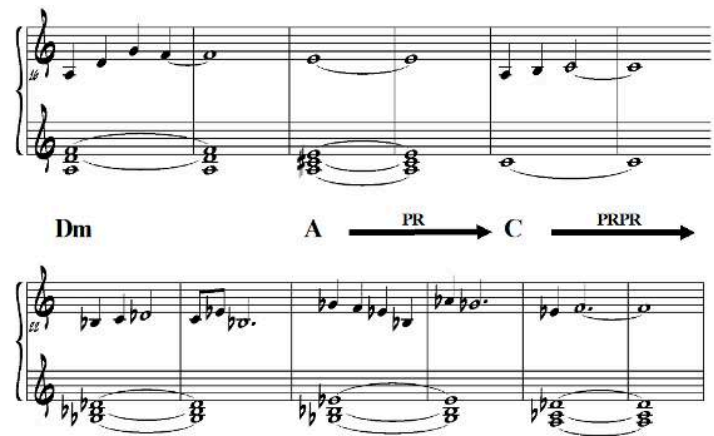

$\mathrm{Gb} \stackrel{\mathrm{R}}{\longrightarrow} \mathbf{E}$ b m $\quad$ D b

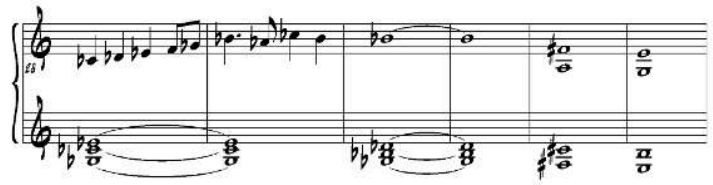

$\mathrm{Cb} \quad \mathrm{Gb} \stackrel{\mathrm{p}}{\longrightarrow} \mathrm{FHm}$ Em

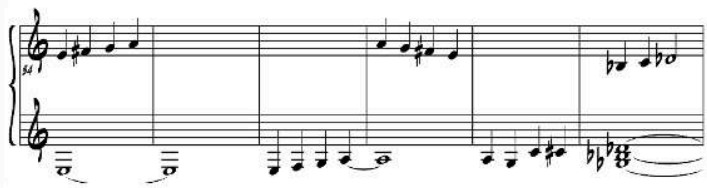

G b

Enchaînements harmoniques de Warszawa

\section{Berlin et l'Europe : le changement de monde référentiel}

Ainsi qu'on a pu l'entrevoir, le changement de monde référentiel du mouvement rock, comme identité collective, et de Bowie, comme identité individuelle, opèrent une conjonction au milieu des années 1970. La fascination pour les univers noirs et carcérau ${ }^{25}$, les prises de position et les références de l'artiste durant la tournée qui les emmène, lui et Iggy Pop, de ville en ville dans une Mercedes décapotable, sont davantage l'expression d'une dérision postmoderniste que d'une réelle adhésion à une idéologie politique clairement affirmée.

Depuis mes années de teenager, j'étais obsédé par l'œuvre émotionnelle pétrie d'angoisse des expressionnistes, des artistes comme des cinéastes, et Berlin avait été leur patrie spirituelle. C'était l'essence du mouvement Die Brücke, de Max Rheinhardt, de Brecht, d'où Metropolis et Caligari tirent leur origine. C'était une forme artistique fondée sur le reflet de l'ambiance et non de l'événement. C'est dans cette direction que je sentais que mon œuvre menait ${ }^{26}$.

L'imagerie choisie par Bowie n'est d'ailleurs pas entièrement nouvelle : d'une certaine manière, elle s'inscrit dans la continuité du personnage schizophrénique du Thin White Duke, sorte de dandy d'apparence sinon germanique, au moins nord-européenne, tiraillé entre un monde existant et matérialiste précipité dans le chaos et des aspirations romantiques d'un idéal fantastique et d'une passion comme fin en soi. Il s'agit plutôt d'appropriations nostalgiques d'époques, souvent très différentes, que cette génération née au lendemain de la guerre, n'a pas connues, et dont elle hérite par l'imagerie collective et une perception faussée par le temps. En décontextualisant les références au passé expressionniste et post-romantique de la ville, les mouvements 
intellectuels et artistiques berlinois de la fin du $\mathrm{xx}^{\mathrm{e}}$ siècle s'approprient une part de leur héritage culturel. Les habitants et les voyageurs de passage à Berlin font vivre cet héritage en le rendant contemporain. Bowie est captivé par l'architecture de la ville : il s'installe dans un appartement du XIX ${ }^{e}$ siècle, dans la rue Haupstrasse du quartier de Schöneberg. L'artiste se passionne pour l'art expressionniste de Die Brücke et s'immerge dans le monde nocturne du cabaret Überbrettl.

Ils retrouvent l'artiste de cabaret Römy Haag, rencontrée lors du concert berlinois de la tournée précédente, qui les initie aux bars et clubs de la ville et, plus généralement, à sa culture bigarrée, entre marginaux, rescapés et immigrés. Bowie, qui abandonne les sophistications vestimentaires et physiques des années passées, opte pour une chevelure blonde naturelle, de simples chemises et jeans, et se ressource lors de régulières promenades pédestres et cyclistes en compagnie de Visconti ${ }^{27}$.

21 Cependant, si le modèle référentiel manifeste un changement de paradigme, ce n'est pas tant dû aux effets évocateurs de cette imagerie, - le rock utilise énormément ce type de procédés -, mais plutôt aux causes profondes générant cette utilisation. En effet, le changement de modèle référentiel est sous-tendu par quelques idées fortes largement puisées dans l'histoire riche et complexe de la ville de Berlin.

Dans un premier temps, le caractère bipolaire de la ville résultant de son histoire entre oppresseur (Époque Wilhelmienne, Troisième Reich) et oppressé (Weimar, Époque de l'Occupation des alliés et Soviétiques) fait écho à l'expérience de l'artiste happé dans une lutte effrénée entre deux attitudes contradictoires, celle du créateur auteur de sa vie et celle de la victime de sa propre addiction à l'héroïne, tiraillé entre passé et présent : la ville, coupée en deux par le mur, effectue, durant cet automne 1977, un retour sur son passé comme en témoignent de nombreuses expositions, dont la célèbre Tendenzen der Zwanziger Jahre (Gropius Bau, Neue Nationalgalerie, Akademie der Künste, Schloss Charlottenburg Orangerie) sur les tendances artistiques des années 1900-1920. Bowie s'exprime sur son rapport à la ville :

Et je parvenais toujours à une étape, lorsqu'il n'y avait plus aucune friction entre la ville et moi. Cela devenait nostalgique, vaguement décadent et je quittais la ville pour une autre. En ce moment, je suis incapable de composer à Los Angeles, New York, ni à Londres, ni à Paris. Il y a quelque chose qui manque. Berlin possède la capacité de faire uniquement écrire les choses importantes - tu ne mentionnes rien d'autre, tu restes silencieux et n'écris rien... et à la fin, tu crées Low ${ }^{28}$.

L'identification entre l'homme et la ville semble favoriser l'imprégnation réciproque de leurs histoires et de leurs destinées. La grandeur, puis la chute de la ville, constituent une métaphore de la propre histoire de l'artiste. Bowie recherche dans ce nouveau lieu, l'anonymat nécessaire pour se guérir de sa paranoïa.

Dans un deuxième temps, les références au Bauhaus non ornemental de l'architecte Adolf $\operatorname{Loos}^{29}$, qui a légué à Berlin sa physionomie de l'époque de Weimar, intéressent Bowie au plus haut point: il déménage d'ailleurs dans un immeuble Altbau de la Kurfürstenstrasse, dont la façade grise est caractéristique d'une esthétique fuyant le glamour ou les paillettes de la Berlin Fin de Siècle pour le dépouillement fonctionnel. Selon Rüther, « Bowie est autant fasciné par le caractère dégradé et anonyme des blocs d'immeubles sociaux que par les vestiges de la grandeur impériale $»^{30}$.

Dans un troisième temps, l'immersion de l'artiste dans la culture expressionniste de la ville est profonde: dans le malaise social consécutif aux bouleversements culturels $\mathrm{du}$ passé, Bowie perçoit le reflet d'une difficile définition de son identité sexuelle. Il 
parcourt les musées et les expositions du groupe Die Brücke des années 1900-1910, fait la rencontre de la toile de Erich Heckel, Roquairol, représentant Ludwig Kirschner, sur le front de la guerre des tranchées (1917) en proie à une dépression, et qui servira de modèle à la pochette de 'Heroes'. Il peint même: Man with a Red Box (1976) dans un style proche des artistes de Die Brücke ou d'Egon Schiele, auquel il envisageait de consacrer un film avec Charlotte Rampling en 1978. Thobias Rüther convoque la pensée du philosophe marxiste Ernst Bloch, pour décrire les processus à l'œuvre dans l'effondrement de la culture bourgeoise de l'entre-deux-guerres et l'émergence de l'expérimentation avant-gardiste en Allemagne. Il ajoute que :

Durant ce temps, Bowie vient s'établir à Berlin pour mener ses expériences, quarante ans plus tard, la vacuité avait augmenté. Les Berlinois vivent leur quotidien dans une crise, mais leur ville est un remarquable patchwork des différentes périodes à travers lesquelles Bowie erre dans ses rêves éveillés ${ }^{31}$.

La période de Berlin ouvre, pour Bowie comme pour la culture pop, une ère nouvelle où références au passé et au présent se mêlent et se répondent en écho, démontrant que l'histoire semble se répéter, et qu'à l'effondrement, en 1920, de la culture académique européenne du début du siècle répond celle, en 1970, de la culture américaine de masse.

Enfin, Bowie s'approprie la tradition du Gesamtkunstwerk (art total) de la ville, véritable expression d'une Weltanschauung (conception du monde) qui se manifeste dans la peinture, la musique, la littérature, l'architecture, le théâtre et le cinéma: Bowie reconnaît dans l'effervescence contemporaine artistique de la ville, ainsi que dans l'héritage du passé, des problématiques qui sont aussi les siennes. Il souhaite être un pont entre les expressions artistiques.

Je suis devenu une rock star. C'est ce que je fais. Ce n'est pas ma vie entière [...]. J'aurais pu être un peintre. Je voulais être ce genre d'artiste. Je souhaitais m'exprimer dans une sphère artistique. Je ne pense pas que j'étais un bon peintre, alors je suis venu à la musique ${ }^{32}$.

Bowie s'installe à Berlin pendant près de deux ans entre l'été 1976 et le printemps 1978. Selon Rüther, "Pour Bowie, l'histoire de l'Europe est davantage localisée dans sa propre tête que dans un atlas, l'homme malade de la Mitteleuropa, une double personnalité aux membres atrophiés et aux traits totalitaires $»^{33}$. Il erre dans la ville, tel Ulrich, l'homme sans qualités du roman de Robert Musil, réfléchissant sur la confrontation de son identité au monde :

Chaque fois que ses voyages le conduisaient dans des villes nouvelles, auxquelles nulle affaire ne le rattachait, il goûtait profondément le sentiment de solitude qui en naissait, et jamais le sentiment n'avait été aussi fort. [...] Un tel état peut éveiller en celui qui flâne ainsi refermé sur lui-même, le sentiment d'être devenu presqu'un asocial ou un criminel ${ }^{34}$.

Bowie se nourrit ainsi de la ville, et recrée sa propre identité, en y puisant l'essence collective. Il écrit : «Berlin a été ma clinique. Elle m’a ramené au contact des gens [...] Mais, alors même que j'étais sobre, j'avais encore des vertiges durant des jours. Il m'a fallu deux ans pour purifier mon système $»^{35}$. 


\title{
Berlin, 1970 - Vienne, 1870 : le malaise de la fin de siècle
}

\author{
ce complexe anthropologique nommé Romantisme, Michaël Löwy et Robert Sayre \\ soutiennent qu'
}

à partir des années 1970, se développe une réaction croissante contre l'esprit de 68 : divers gauchistes repentis piétinent allègrement leurs idoles idéologiques de naguère [...] découvrent que le paradis existe déjà, et se mettent à chanter les louanges de la modernité dans tous ses aspects : le libéralisme, la logique du droit et de la politique moderne ${ }^{36}$.

31 La remise en cause, voire le rejet, de la modernité mécanique et du progrès matériel au profit d'une organicité perdue constitue un des thèmes récurrents reliant les expériences de l'homme contemporain des $\mathrm{XIX}^{\mathrm{e}}$ et $\mathrm{Xx}^{\mathrm{e}}$ siècles. Les penseurs romantiques considèrent que ce lien nous rattachant à une forme de transcendance, inscrite dans la condition de l'homme primitif et l'organisation de la société traditionnelle, a été progressivement rompu par la société bourgeoise, urbaine et marchande. La recherche d'ailleurs exotiques, temporels et oniriques et d'univers esthétisés devient, dès lors, une compensation à cette perte et à l'impossibilité de modifier le cours de l'histoire et du progrès.

Cette démission face à l'idéal romantique, face à la transformation possible de la société en un monde plus organique, respectueux de la transcendance et de la spiritualité, génère une désillusion culturelle et sociale. Le punk et la cold wave émergent consécutivement à la perte de cette foi, dans un contexte d'accélération d'une mondialisation violente, dans une déstructuration de la société de consommation occidentale et une montée du néo-libéralisme.

D'une certaine manière, la situation de Berlin et de notre monde postmoderne des années 1970 présente de nombreuses analogies avec celle de Vienne qui, un siècle plus tôt, secoua l'ensemble de la Mitteleuropa, entraînant des conséquences culturelles et artistiques similaires. C'est probablement ce malaise fin de siècle, annonciateur d'une mutation sociale de grande envergure, qui fait écho au drame personnel de Bowie et interpelle l'artiste de manière plus ou moins consciente, et le fait se tourner spontanément vers le Romantisme, l'Expressionnisme et le Bauhaus: ces trois esthétiques constituent des manifestations de l'angoisse générée par les changements sociaux et culturels considérables qui font émerger le monde moderne du monde d'hier et des vieux empires dynastiques.

sur l'identité autrichienne naissante dans le bas Empire des Habsburg ${ }^{37}$, Michel Pollack décrit parfaitement les conditions qui ont sous-tendu l'émergence simultanée des esthétiques progressistes dans la ville de Vienne à la charnière des $\mathrm{XIX}^{e}$ et $\mathrm{Xx}^{e}$ siècles. Selon l'auteur de cette étude, on peut observer, dès le dernier tiers du XIX ${ }^{e}$ siècle, cet enchaînement logique de faits sociaux ${ }^{38}$ :

1- contradiction d'une époque de mutation tiraillée entre appel du futur et repli angoissé vers le passé,

2 - existence d'un contrat entre le pouvoir et une nouvelle génération infantilisée dans une Sécession ou une contre-culture, 


\section{exploitées par Bowie. Si l'on rapproche ces tendances ou attitudes génériques du cas} particulier de la trilogie dite berlinoise, on remarque que :

Nombre de titres empruntent des ailleurs temporels ou géographiques, soit sous la forme du voyage et de sa possibilité d'échapper à la quotidienneté du monde, soit sous celle de l'évocation de l'ailleurs temporel ou géographique, même si cet ailleurs consiste en une partie de la ville ("Warszawa », "Subterraneans ", " Moss Garden », « Neuköln », « The Secret Life of Arabia », « Fantastic Voyage », « African Night Flight », etc.).

49 2- La ville en devenir qu'est Berlin fait écho au devenir de la personnalité du créateur et constitue un territoire propice aux expérimentations. «Pour Bowie, la ville est un médium. Ich und die Stadt (Moi et la ville) [...]. Il n'y a plus de différence entre l'intérieur et l'extérieur, tout est identitaire, l'être humain et les murs croissant l'un à l'intérieur de l'autre $\star^{40}$.

50 3- Nombre de titres des trois albums berlinois constituent des productions musicales fortement esthétisées, échappant de manière volontaire aux canons habituels du rock de l'époque ("A New Career in a New Town », «Warszawa », " Art Decade », « Weeping Wall », « V2 Schneider », « Sense of Doubt », « Moss Garden », « Neuköln », « The Secret 
Life of Arabia »). En outre, «Art Decade» (Low) et «DJ» (Lodger) sont les deux seuls titres dont les textes ou l'argument extramusical se réfèrent à l'autonomie de l'art.

51 4- La tendance à abandonner, dans la musique rock, un format stéréotypé d'écriture musicale pour un mode de composition, renouvelé pour chaque œuvre, empruntant des univers complexes et multiples, - improvisation, minimalisme, nouveaux instruments électroniques - peut être considéré comme une évolution vers un processus de création plus naturel et individualisé.

Bowie, mais aussi Eno évidemment, adopte le studio d'enregistrement comme un terrain d'expérimentation, de collages et de montage. Déjà entrevue durant les sessions de Station to Station, sa démarche consiste maintenant à rentrer en studio sans aucune chanson composée. C'est du studio et de ses possibilités que naissent les idées musicales ${ }^{41}$.

52 5- Dans le prolongement d'un retour vers une composition plus organique, le concept d'art total transforme en profondeur les liens existant entre expressions artistiques : la relation de subordination du texte à la musique ou de la musique au texte est abandonnée au profit du cadre plus large d'une transversalité d'intertextuelle.

Bowie appelle ce qu'il fait “ écrire " et non « composer ». Pour lui, c'est davantage un état permanent qu'un acte intentionnel [...] Vous voulez dire « écrire » M. Bowie, comme écrire des livres ? Et Bowie répondait, de manière provocatrice, que c'est exactement ce qu'il faisait, qu'il travaillait sur une collection d'histoires courtes. ${ }^{42}$

\section{Low-'Heroes'-Lodger : figures culturelles, figures musicales}

53 C'est au mois d'août 1976 que commence, au Château d'Hérouville (Vexin français), l'enregistrement de Low. L'univers sonore du LP fait rupture avec celui des productions précédentes: il constitue une hybridation du monde sonore du Krautrock et des expérimentations électroniques de Brian Eno. Les deux autres albums de la trilogie suivront à distance d'un an pour 'Heroes' (septembre 1977), puis d'un an et demi pour Lodger (mai 1979). Au printemps 1979, Bowie avait déjà quitté Berlin depuis un peu plus d'un an. On peut toutefois reconnaître que l'unité stylistique musicale et littéraire des trois albums de la trilogie est réelle. Pour s'en convaincre, il suffit de répartir leurs 31 titres sur les 9 figures culturelles caractéristiques des problématiques développées par la confrontation de l'histoire de l'artiste à l'histoire de la ville. Ces figures culturelles ou thématiques sont déclinées ci-dessous :

le voyage ; le rêve ; l'art ; l'ailleurs ; la condition humaine ; l'altérité ; l'introspection ; l'autodestruction ; l'identité sexuelle.

Les tableaux suivants montrent comment ces thématiques se répartissent au fil des titres et les albums. On constate que Low constitue l'album le plus équilibré du point de vue des thématiques évoquées par les textes, alors que 'Heroes' se concentre davantage sur celles de l'ailleurs et de l'identité et Lodger sur celles du voyage, du monde onirique, et des phénomènes identitaires. Sur l'ensemble des titres, l'harmonie de tierces, souvent avec changement de mode, affecte, à différentes échelles, neuf sur dix des titres de Low (" Speed of life ", "Breaking glass », " What in the world ", "Always Crashing in the same car", "Be my wife ", "A new career in a new town", «Warszawa », « Art Decade», « Weeping Wall»), et 7 sur 10 de 'Heroes' (« Beauty and the Beast ", "Joe the lion ", "Sons of the Silent Age ", "V2-Schneider ", "Sense of 
Doubt ", « Neuköln », "The Secret Life of Arabia »). Même si elle constitue un trait distinctif de la couleur musicale de la discographie berlinoise de Bowie, elle ne semble toutefois pas corrélée à une figure culturelle particulière.

Fig. 6

\begin{tabular}{|c|c|c|c|}
\hline Figures Culturelles & LOW & HEROES & LODGER \\
\hline Enthousiasme du voyage & $\begin{array}{c}\text { A New Career in a New } \\
\text { Town }\end{array}$ & & $\begin{array}{c}\text { Fantastic Voyage } \\
\text { African Night Flight } \\
\text { Move on } \\
\text { Red Sails }\end{array}$ \\
\hline $\begin{array}{c}\text { Monde onirique, Temps } \\
\text { immobile }\end{array}$ & $\begin{array}{c}\text { Warszawa } \\
\text { Subterraneans }\end{array}$ & & $\begin{array}{c}\text { Yassassin } \\
\text { Look back in Anger }\end{array}$ \\
\hline Autonomie de l'art & Art Decade & & DJ \\
\hline $\begin{array}{l}\text { Ailleurs temporel et } \\
\text { géographique }\end{array}$ & $\begin{array}{c}\text { Warszawa } \\
\text { Weeping Wall } \\
\text { Subterraneans }\end{array}$ & $\begin{array}{c}\text { Sons of the Silent Age } \\
\text { V2 Schneider } \\
\text { Moss Garden } \\
\text { Neuköln } \\
\text { The Secret Life of Arabia }\end{array}$ & \\
\hline $\begin{array}{l}\text { Altérité, Intérieur / } \\
\text { Extérieur }\end{array}$ & Be my Wife & $\begin{array}{c}\text { Beauty and the Beast } \\
\text { Heroes }\end{array}$ & \\
\hline Condition humaine & Always Crashing in the Car & Sense of Doubt & \\
\hline Introspection psychologique & $\begin{array}{l}\text { Breaking Glass } \\
\text { What in the World } \\
\text { Sound and Vision }\end{array}$ & & Red Money \\
\hline Autodestruction & & $\begin{array}{l}\text { Joe the Lion } \\
\text { Blackout }\end{array}$ & \\
\hline Identité, Lutte sexuelle & & & $\begin{array}{c}\text { Boys keep swinging } \\
\text { Repetition }\end{array}$ \\
\hline
\end{tabular}

Répartition des figures culturelles sur la trilogie

\section{Conclusion}

À Berlin, la collaboration de David Bowie et de Brian Eno, entre 1976 et 1979, crée un nouveau type d'instrumental et de chanson, sous-tendu par un processus de création organiciste et d'art total, nourri de références à des ailleurs temporels et géographiques, et caractérisé par la recherche de couleurs harmoniques, ainsi que par un certain statisme hypnotique et synthétique : le paysage sonore romantique. Cette collaboration ouvre la voie à de nouvelles hybridations expérimentales fécondes pour le rock, et réinstaure les enchaînements harmoniques hérités du Romantisme dans l'univers du rock jusqu'alors régi par les dérivés de l'harmonie du blues. Ainsi que le soutient Mathieu Thibault,

Bowie incarne l'artiste postmoderne par excellence [car] il brise également les barrières hiérarchiques entre les arts, les disciplines et l'opposition désuète des domaines savants et populaires. La personnalité de l'artiste, formidable catalyseur d'influences, dépasse la simple somme de références en les intégrant à un style personnel et inclassable ${ }^{43}$.

Durant ses années berlinoises, David Bowie apparaît comme une figure du néoromantisme, véritable révélateur de l'angoisse générée par les mutations du monde post-industriel. En puisant ses références parmi l'art et la culture de la ville et, au-delà, d'une certaine Mitteleuropa du début du $\mathrm{xx}^{\mathrm{e}}$ siècle, il jette un pont entre le malaise d'une époque traumatisée par le premier conflit mondial et l'effondrement de la civilisation 
occidentale d'une part, et la désillusion des excès matérialistes de la mécanisation, de la consommation et du star system contemporains d'autre part.

Cependant, Bowie n'use pas de ces références de manière directe, c'est-à-dire en les manipulant comme des images n'affectant sa création que de manière plus ou moins superficielle. Il les intègre en en récupérant l'essence qu'il réinvestit au cœur d'un nouveau matériau, fruit d'une intense collaboration avec Brian Eno. À travers cette réutilisation, comme substance, du passé glorieux ainsi que du passé plus sombre de la Mitteleuropa, le reflet iconographique d'une ville et de sa culture s'efface au profit des attitudes, des perceptions et des processus à l'origine de l'art. C'est probablement en ce sens qu'il faut comprendre l'héritage berlinois et romantique de David Bowie.

Bloch, Ernst. Erbschaft dieser Zeit, 1935, Héritage de ce temps. Jean Lacoste trans. Paris :

Klincksiek, 2017.

Bowie, David. Uncut interview : David Bowie on Berlin - the real "uncut" version. <http://

www.bowiewonderworld.com/features/dbuncut.htm>, dernière consultation du site 15 août 2017.

Cohn, Richard. Audacious Euphony: Chromaticism and the Triad's Second Nature. New York: Oxford University Press, 2012.

Löwy, Michael. Sayre, Robert. Révolte et mélancolie, Le romantisme à contre-courant de la modernité. Paris : Payot, 1992.

Musil, Robert. Der Mann ohne Eigenschaften, 1932, L'homme sans qualités. Philippe Jaccottet trans. tome 2. Paris : Seuil, 1956.

olive, Jean-Paul. Musique et montage, essai sur le matériau musical au début du $\mathrm{XX}^{e}$ siècle.

Paris : L'Harmattan, 1998.

Pollack, Michel. Vienne 1900 : une identité blessée. Paris : Gallimard, 1992.

Rüther, Thobias. Heroes, David Bowie and Berlin. London: Reaktion Books, 2014.

Thibault, Mathieu. La trilogie Bowie-Eno : influence de l'Allemagne et de Brian Eno sur les albums de David Bowie entre 1976 et 1979. Paris : Camion Blanc, 2011.

Thibault, Mathieu. David Bowie : l'avant-garde pop. Paris : Le Mot et le Reste, 2013.

\section{NOTES}

1. Thibault, Mathieu. David Bowie : l'avant-garde pop, Paris: Le Mot et le Reste, 2013: 7.

2. Thibault, Mathieu. Op. Cit.

3. Selon la terminologie de Walter Benjamin, voir Sens unique, recueil de textes, un "photomontage d'un voyage à travers l'époque ", cité par olive, Jean-Paul. Musique et montage, essai sur le matériau musical au début du XXe siècle, Paris: L'Harmattan, 1998.

4. Thibault, Mathieu. Op. Cit.: 178-179.

5. "When I left LA I discovered how little I knew, how little I had to say. The lack of lyrics on Low reflects that I was literally stuck for words", Rüther, Thobias. Heroes, David Bowie and Berlin, London: Reaktion Books, 2014: 36.

6. “No, no, no...I'm not Pierrot. I'm Everyone. What I'm doing is theatre, and only theatre. I'm using myself as a canvas and trying to paint the truth of our time on it. The white face, the baggy 
pants - they're Pierrot, the eternal clown putting over the great sadness of 1976", Rüther, Thobias. Op. Cit.: 36.

7. Le terme est une référence au concept freudien. Voir Freud, Sigmund, Das Umheiliche, trad. Française, L'inquiétante étrangeté, Paris, Folio Essais, 1988.

8. "In 1975 and 1976 he is mad on listening to Germans bands [...] Bowie is again free to concentrate solely on what he is really interested in: art. American funk and soul are out [...] He is fed up with L.A., with America, with rock'n roll [...] German electronic music is the most radical thing that pop music has to offer in this period", Rüther, Thobias. Op. Cit.: 37.

9. Les albums Low, Heroes, et Lodger, sortis entre 1976 et 1979 sont souvent considérés comme une trilogie, que l'on nomme berlinoise, même si Bowie avait déjà quitté Berlin avant la sortie du dernier album.

10. Le terme "sème» est utilisé ici dans le sens d'une unité de signification culturelle, susceptible de produire des réalisations aptes à communiquer une expérience humaine.

11. Kraftwerk est un groupe de la scène alternative électronique allemande. Leur quatrième album, Autobahn, est sorti à l'automne 1974, sur le label Philips.

12. "their compositions were well prepared and honed before entering the studio. My work tended to expressionist mood pieces, the protagonist (myself) abandoning himself to the 'zeitgeist' (a popular word at the time), with little or no control over his life. The music was spontaneous for the most part and created in the studio [...] What I was passionate about in relation to Kraftwerk was their singular determination to stand apart from stereotypical American chord sequences and their wholehearted embrace of a European sensibility displayed through their music. This was their very important influence on me", Bowie, David. Uncut interview: David Bowie on Berlin - the real "uncut" version, <http://www.bowiewonderworld.com/ features/dbuncut.htm>, dernière consultation le 15 août 2017.

13. Neu! Kraftwerk est un groupe de la scène krautrock allemande, né de la scission de quelques uns des membres de Kraftwerk (Klaus Dinger, Michael Rother), en 1971.

14. "I bought my first vinyl Neu 2 in Berlin around 1975 while I was on a brief visit [...] I bought it because that they were a spin off $\mathrm{K}$ and had to be worth hearing. Indeed, they were to prove to be K's wayward anarchistic brothers. I was completely seduces by the setting off the aggressive guitar-drone against the almost-but-not-quiet robotic/machine drumming of Dinger. Although fairly tenuous, you can hear a little of their influence on the track Station to Station", Rüther, Thobias. Op. Cit.: 41.

15. "Both Bowie and Kraftwerk conceived of their act as a whole - the music, the clothes, the artwork [...] all integrated and self referring [...] both nurtured a camp sensibility working the delicate seam that lies between irony and earnestness. Both blended postmodern pastiche with a retromodernist aesthetic. Both made emotional music by seeming to negate emotion. Seen through the prism of psychiatry, the work of both comes across as rather autistic", Rüther, Thobias. Op. Cit.: 40.

16. Les théories néo-riemanniennes, dont l'ouvrage de David Lewin « Generalized Musical Intervals and Transformations", publié en 1987, constitue probablement l'un des actes fondateurs, considèrent, dans l'héritage de Hugo Riemann, que les collections diatoniques, comme les triades, deviennent le produit de transformations autonomes sélectionnées et répertoriées pour leurs vertus symétriques, formalisables via un modèle. Elles s'articulent autour du concept de transformation d'unités harmoniques simples, les triades majeures et mineures ou les accords de septième, via une conduite parcimonieuse des voix favorisant les notes communes et obéissant à la loi du plus court chemin. Elles définissent ces transformations au sein d'un système dualiste fondé sur l'équivalence enharmonique.

17. Les signes + et - désignent la direction ascendante ou descendante dans laquelle s'opère le déplacement chromatique entre deux accords. 
18. "Chromatic progressions of triads excited the Romantic imagination not because they conformed to expectations about triadic behavior and succession but because they confounded them [...]. Even in nonprogrammatic, instrumental music, they are capable of evoking the strange, magical, and inscrutable", Cohn, Richard. Audacious Euphony: Chromaticism and the Triad's Second Nature, New York: Oxford University Press, 2012: X.

19. «Space Oddity » est une chanson extraite de l'album éponyme de David Bowie, sorti en 1969.

20. «Scary Monsters and Super Creeps » est une chanson extraite de l'album éponyme de David Bowie, sorti en 1980.

21. Le terme de Cold Wave britannique fait référence à une esthétique des années 1980, dont les groupes représentatifs sont Joy Division, Bauhaus, Ultravox...

22. Thibault, Mathieu. Op. Cit.: 180.

23. "Eno wants to fuse this strange, rigid, stiff-stuff of Kraftwerk with the weird physical feeling of the American funk band Parliament. When you find someone with the same problems, Eno says, you tend to become friendly with them, Rüther, Thobias. Op. Cit.: 43.

24. «Warszawa » est un titre extrait de l'album Low de David Bowie, sorti en 1977.

25. "I want tanks, turbines, smokestacks, fluorescent lightening, alleyways, cages, watchtowers, girders, beams, Albert Speer", Rüther, Thobias. Op. Cit.: 48-50.

26. "Since my teenage years, I had obsessed on the angst ridden, emotional work of the expressionists, both artists and film makers, and Berlin had been their spiritual home. This was the nub of Die Brücke movement, Max Rheinhardt, Brecht and where Metropolis and Caligari had originated. It was an art form that mirrored lie not by event but by mood. This was where I felt my work was going", Bowie, David. Uncut interview: Op. Cit.

27. Thibault, Mathieu. Op. Cit.: 179-180.

28. "And I've always got to the stage where there isn't any friction between a city and me. That became nostalgic, vaguely decadent and I left for another city. At the moment, I'm incapable of composing in Los Angeles, New York or in London or Paris. There is something missing. Berlin has the ability to make you write only the important things - anything else you don't mention, you remain silent, and write nothing... and in the end, you produce Low", Rüther, Thobias. Op. Cit.: 79.

29. À ce propos, on peut consulter l'ouvrage d'Adolf Loos, - «D'un combat de trente années, je suis sorti vainqueur. J'ai libéré l'humanité de l'ornement superflu», Loos, Adolf. Ornement et crime, traduit de l'allemand, Paris, Payot et Rivages, 2003.

30. "Bowie is equally fascinated by the rundown, anonymous quality of the social housing blocks and by the vestiges of imperial grandeur", Rüther, Thobias. Op. Cit.: 64.

31. "By the time, Bowie comes to Berlin to experiment 40 years later, the vacuum has only increased. Berliners live their contemporaneously with crisis, but their city is a remarkable jumble of different periods that Bowie wanders through in his waking dream", Rüther, Thobias. Op. Cit.: 83.

32. "I became a rock star. It's what I do. It's not my whole life [...] I could have been a painter. I wanted to be some kind of artist. I wanted to prove myself in some field as an artist. I didn't think I was a very good painter, so I went into music", Rüther, Thobias. Op. Cit.: 113.

33. "For Bowie, the history of Europe is located more in his own head than in the atlas, the sick man of Mitteleuropa, a split personality with withered limbs and totalitarian features ", Rüther, Thobias. Op. Cit.: 54-55.

34. Musil, Robert. Der Mann ohne Eigenschaften, 1932, L'homme sans qualités, trad. de Philippe Jaccottet, tome 2, Paris: Seuil, 1956: 73-74.

35. "Berlin was my clinic. It brought me back in general touch with people [...] But I would still have days when things were moving round the room even when I was sober. It took two years to cleanse my system", Rüther, Thobias. Op. Cit.:165. 
36. Löwy Michael. Sayre Robert. Révolte et mélancolie, Le romantisme à contre-courant de la modernité, Paris: Payot, 1992: 284.

37. Pollack, Michel. Vienne 1900 : une identité blessée, Paris: Gallimard, 1992.

38. Pollack, Michel. Op. Cit., voir le chapitre 2, « Le prix de la modernisation »: 47-74.

39. Pollack, Michel. Op. Cit., voir le chapitre 4, «Jung-Wien, de l'identité perdue à l'art pur»: 111-151.

40. "For Bowie, the city is the medium. „Ich und die Stadt" [...] There is no longer any difference between the interior and the exterior, everything is identical, the human being and walls growing into one another", Rüther, Thobias. Op. Cit.: 146.

41. Thibault, Mathieu. La trilogie Bowie-Eno : influence de l'Allemagne et de Brian Eno sur les albums de David Bowie entre 1976 et 1979, Paris: Camion Blanc, 2011.

42. "Bowie calls what he is doing writing, not composing. For him, it's more like a permanent state rather than a conscious act [...] You mean writing, Mr. Bowie, like writing books? And Bowie provocatively replies, that's exactly what he is doing, he is working on a collection of short stories, Rüther, Thobias. Op. Cit.: 125.

43. Thibault, Mathieu. Op. Cit.: 7-8.

\section{RÉSUMÉS}

En raison de sa richesse intermédiatique, l'univers artistique de David Bowie offre un lieu privilégié pour l'exploration du lien entre sonore et symbolique. Même si ce lien est présent tout au long de la carrière de l'artiste, c'est plus précisément sur sa période dite berlinoise que cet article propose de se pencher. Parce que les années 1976-1979 constituent une rupture esthétique dans l'évolution artistique de Bowie, et plus généralement dans l'histoire du rock et l'émergence de la cold wave, parce que ces années pourraient être comparées à une sorte de phase néoromantique tournée vers l'univers expérimental de la musique électronique germanique la Vienne fin de siècle de Bowie -, et parce que cette période se nourrit, sous l'impulsion de Brian Eno, d'influences musicales et artistiques, passées et contemporaines, la trilogie Low-'Heroes'Lodger occupe une place de choix dans l'univers du créateur. L'ailleurs exotique, le temps immobile, l'étrangeté onirique, l'ambiguïté sexuelle, la déchirure entre l'être intérieur et l'être social, le formalisme esthétisé et le recours à l'organicisme constituent, tant pour Bowie que pour la ville qui l'accueille, autant de figures anthropologiques induites par le contexte historique et social de parcours individuels et collectifs.

Because of its cross-media resourcefulness, the artistic universe of David Bowie offers a first-rate basis to explore the link between sonority and symbolism. Even if this link is to be found everywhere all along the artist's career, this article will focus on the so called Berliner period. Because the years 1976 to 1979 make up an aesthetic break in Bowie's artistic evolution, and more generally in the history of rock music with the emergence of cold wave music; because those years could be compared with a kind of neo-romantic phase focused on the experimental universe of German electronic music - Bowie's 'turn-of-the-century Vienna' -; what more because this period is fraught with musical and artistic influences, in the pass as well as in the present. Under the impulse given by Brian Eno, the 'Low-'Heroes'-Lodger' trilogy always has a favorite place in the creator's universe. A geographic and historical exoticism, a static perception of time, a dreamlike strangeness, a sexual bivalence, the torn gap between the interior being and 
its social counterpart, an anesthetized formalism and the recourse to organicism make up, as much to Bowie as to the incoming city, so many anthropological figures induced by the historical and social context of individual and collective lives.

\section{INDEX}

Keywords : Bauhaus, Berlin, Bowie, Cold wave, Expressionism, Gesamtkunstwerk, Krautrock, Electronic Landscape, Romanticism

Mots-clés : Bauhaus, Berlin, Bowie, Cold wave, Expressionnisme, Gesamtkunstwerk, Krautrock, Paysage électronique, Romantisme

\section{AUTEURS}

\section{HUGUES SERESS}

Professeur de Culture et d'Analyse Musicale - Chercheur associé Institut de Recherche en Musicologie (IReMus), UMR 8223 - Centre d'Etudes Supérieures de Musique et de Danse de Poitiers

contact@seress-hugues.hu 